Case Reports

\title{
Prosthetic Valve Endocarditis Due to Clostridium Limosum: A Case Report and Review of the Literature
}

\author{
${ }^{1}$ Lok Yung, ${ }^{1,2}$ Carl Urban, ${ }^{3}$ Negin Niknam and ${ }^{1}$ Glenn Turett \\ ${ }^{I}$ The James J Rahal Jr Division of Infectious Diseases, New York Presbyterian Queens, United States \\ ${ }^{2}$ Weill Cornell Medical College, Cornell University, New York, United States \\ ${ }^{3}$ Department of Infectious Diseases, Northwell Health, Manhasset, New York, United States
}

\author{
Article history \\ Received: 21-08-2019 \\ Revised: 30-09-2019 \\ Accepted: 10-10-2019 \\ Corresponding Author: \\ Carl Urban \\ The James J Rahal Jr Division of \\ Infectious Diseases, New York \\ Presbyterian Queens, United States \\ Email: cmurban@nyp.org
}

\begin{abstract}
Clostridium limosum is an anaerobic gram positive rod-shaped bacterium found in soil and can cause infections in a variety of animals. Rarely has this organism caused infections in humans. While clostridial prosthetic valve endocarditis is reported infrequently, we document the first human case of prosthetic valve endocarditis as the sole pathogen, due to Clostrdium limosum.
\end{abstract}

Keywords: Clostrdium limosum, Prosthetic Valve Endocarditis, Management

\section{Introduction}

Clostridium limosum is an anaerobic gram positive rodshaped bacterium found in soil and can cause infections in various animals including humans, cattle, alligators, chickens, dogs and farmed minks (Cato et al., 1948; Bistrom et al., 2016; Shibuya et al., 1994; Gordon and Axelrod, 1985; Gorbach and Thadepalli, 1975). It has also been isolated from home-preserved meat and human clinical specimens including blood, peritoneal and pleural fluid, soft tissue and lung tissue (Cato et al., 1948; Gorbach and Thadepalli, 1975). Clostridium limosum was reported to be susceptible to chloramphenicol, erythromycin, penicillin $\mathrm{G}$, clindamycin and tetracycline in a previous publication (Felner and Dowell, 1970). There are only 3 cases of human infection with this organism described in the English literature, including 1 prosthetic valve infection and 2 infected sacral decubitus ulcers (Gordon and Axelrod, 1985; Shibuya et al., 1994). In each of these patients, $C$. limosum was part of a mixed infection. Clostridial prosthetic valve endocarditis is also a very rare entity, with only 4 previously reported cases (Gordon and Axelrod, 1985; Alvarez-Eicoro and Sifuentes-Osorio, 1984; Robles et al., 1997). We present a case of Clostridium limosum prosthetic valve endocarditis and the first human infection with this organism as the sole pathogen.

\section{Case Presentation}

A 59-year-old man with a history of cardiac and cerebral vascular disease, hypertension and hyperlipidemia, who underwent 2 cardiac stents and a bovine aortic valve replacement in 2013, was brought into our ED by EMS after being found on the street. On examination, he was slightly confused with slurred speech. His temperature was $36.2^{\circ} \mathrm{C}$, pulse 61 beats per minute, blood pressure $162 / 79 \mathrm{~mm} \mathrm{Hg}$ and respiratory rate 15 breaths per minute. His neurological examination showed dysarthria, left facial asymmetry, decreased L-sided muscle tone (upper extremities more than lower extremities) and positive testing for extinction on double simultaneous stimulation.

Complete blood count was significant for a white cell count of $19.5 \mathrm{k}$ per $\mathrm{mm}^{3}$, hemoglobin $11.1 \mathrm{~g} / \mathrm{dL}$ and platelet count of $203 \mathrm{k}$ per $\mathrm{mm}^{3}$. Comprehensive metabolic panel was significant for sodium $140 \mathrm{mEq} / \mathrm{L}$, bicarbonate $24 \mathrm{mEq} / \mathrm{L}$, urea nitrogen $23 \mathrm{mg} / \mathrm{dL}$ and creatinine $1.22 \mathrm{mg} / \mathrm{dL}$. Lactic acid was $0.95 \mathrm{mmol} / \mathrm{L}$. Blood cultures were sent to the clinical microbiology laboratory.

Electrocardiography showed sinus rhythm without ST-segment changes. Computed tomography of the head with contrast findings, were suggestive of occlusion at the proximal A2 segment of the right anterior cerebral artery. Magnetic resonance imaging of the head without contrast was suggestive of a large area of acute to subacute infarct involving the right anterior cerebral artery territory. Possible punctate foci of acute to subacute infarct were also noted within the poster lateral aspects of both parietal lobes, as well as a possible subacute infarct within the lateral aspect of the right cerebellar hemisphere. Old bilateral basal ganglia lacunar infarcts and an old focal area of infarct within 
the left cerebellar hemisphere were also observed. Ultrasound venous Doppler of lower extremities bilaterally were unremarkable. Chest X-ray showed no acute pulmonary disease.

Transesophageal echocardiography revealed a bioprosthesis in the aortic position with a mobile vegetation and moderate eccentric aortic regurgitation. The aortic annulus appeared mildly thickened, representing either post-surgical change or abscess. There was significant pericardial effusion and color Doppler flow demonstrated no obvious evidence of shunt or patent foramen ovale.

\section{Hospital Course}

The patient was admitted to the general medicine floor. Cardiology, Cardiothoracic surgery and Infectious Diseases were consulted. Vancomycin, cefepime and rifampin were administered intravenously for empiric coverage while awaiting blood culture results. Given the patient's recent stroke and risk of hemorrhagic conversion, the lack of further embolic events and no signs nor symptoms of severe congestive heart failure, cardiothoracic surgery recommended not to proceed with surgery.

Blood cultures were initially reported as growing a gram negative rod in both aerobic bottles of 2 sets at 76.8 and 78.4 hours. Metronidazole was added to the antibiotic regimen to cover for possible facultative gram negative anaerobes. The organism was ultimately identified as Clostridium limosum by VITEK-2. Once knowing the identity of this organism, the Infectious Diseases team further questioned the patient about possible soil exposures. He denied gardening or other contact with soil. He does however work as a mechanic for the NYC Department of Transit maintaining their vehicles and this was his likely exposure to soil and this organism. Repeat blood cultures drawn 5 days after starting antibiotics were negative. Antibiotic susceptibilities were determined by e-test methodology which showed a minimum inhibitory concentration of 4 $\mathrm{ug} / \mathrm{ml}$ for clindamycin, $0.25 \mathrm{ug} / \mathrm{ml}$ for ertapenem, 0.19 $\mathrm{ug} / \mathrm{ml}$ for meropenem, $1.0 \mathrm{ug} / \mathrm{ml}$ for cefoxitin, 0.50 $\mathrm{ug} / \mathrm{ml}$ for ampicillin-sulbactam and antibiotic treatment was reassessed accordingly. The patient had a history of an allergy to penicillins 40 years ago when given during a bout of mononucleosis. Allergy and immunology tested the patient and deemed it safe to give him penicillins. Antimicrobial therapy was switched to ampicillin-sulbactam and rifampin. However, 4 days into this treatment he developed new onset fevers, leukocytosis with increasing eosinophilia, rising serum creatinine and a new diffuse macular rash. His antimicrobial therapy was switched to meropenem with continuation of rifampin. The rash, leukocytosis and eosinophilia rapidly resolved.
The patient requested transfer to a nearby institution so he could be cared for by his regular cardiologist. Since he was medically stable, on hospital day 26 he was transferred.

Upon transfer, rifampin was discontinued and meropenem was switched to ertapenem. Metronidazole was also added as an adjunct therapy. A repeat transesophageal echocardiogram showed thickening of the bioprosthetic aortic valve leaflets with a mobile echodensity associated with the non-coronary cusp consistent with a vegetation. Also, mild thickening of the aortic root was noted and an aortic root abscess could not be entirely ruled out.

During the perioperative work up, the patient developed hemorrhagic conversion in the right anterior cerebral artery territory. The valve replacement surgery was postponed as there was high risk of worsening intracranial bleeding and devastating neurological injury during heparinization for cardiopulmonary bypass.

An abdominopelvic CT scan, done to rule out any intraabdominal pathology, showed heterogeneous hepatic parenchyma in the right lobe with possible developing abscess and also multiple tiny hepatic hypodensities suspicious for microabscesses. A repeat CT done ten days later showed sub centimeter hepatic hypodensities likely representing biliary hamartomas.

Five weeks into treatment at the second hospital, the patient underwent aortic valve replacement. The previous prosthesis was removed without complications and of note, purulent material was found in the noncoronary sinus which was sent for culture. Both the valve culture and the non-coronary sinus culture were negative. Despite these negative cultures, the decision was made to extend the antibiotic course for another 6 weeks since there was purulent material in the noncoronary sinus and anaerobic organisms are generally harder to grow. The patient completed this course of treatment uneventfully. After hospital discharge, the plan was for him to follow-up with the Infectious Diseases service and have surveillance cultures done off antibiotics to assure that the infection was truly eradicated. Unfortunately, the patient was lost to follow-up after discharge. Repeated attempts to contact him were unsuccessful.

\section{Discussion}

Clostridium limosum belongs to the family of Clostridiaceae. It is an anaerobic gram positive rod found in soil and can cause infections in various animals (Cato et al., 1948; Bistrom et al., 2016; Shibuya et al., 1994; Gordon and Axelrod, 1985; Gorbach and Thadepalli, 1975). Virulence factors associated with this organism include collagenase, lecithinase and toxins which target monocytes and 
macrophages (Cato et al., 1948; Barth et al., 2015). It is rare for this organism to cause disease in humans and when it does, it is usually part of a polymicrobial infection. Our patient is the fourth described case of human infection with $C$. limosum and the first where this organism was the sole pathogen. $\mathrm{He}$ is also the first patient to survive this infection. One of the 3 prior cases was of prosthetic valve endocarditis with Pseudallescheria boydii also isolated from prosthetic material. That patient died on the second day post valve replacement surgery (Gordon and Axelrod, 1985). Two cases involved infected sacral decubiti leading to gas gangrene where $C$. limosum was one of many organisms isolated. Both of these patients died from their infections (Shibuya et al., 1994). One article discussing isolation of Clostridium spp. in human infections, in a table of isolates from 87 patients with skin and soft-tissue infection, listed C. limosum in two cases but no other information was provided (e.g., patient characteristics, mono or polymicrobial infection, treatment and outcome) (Gorbach and Thadepalli, 1975).

It is likely that our patient acquired $C$. limosum through soil contact in his job maintaining vehicles for the NYC Department of Transportation. It is also likely that his symptoms of a stroke on presentation to the hospital were a manifestation of septic emboli from his underlying endocarditis.

Clostridial prosthetic valve endocarditis is also a very rare entity and there are only four previously described cases: 1. The mixed fungal infection mentioned above in a 52-year-old man with a prosthetic aortic valve; 2. C. perfringens in a 23 -year-old man with prosthetic aortic and mitral valves. He was improving on intravenous penicillin but died 12 days into his hospitalization from a secondary infection (Alvarez-Eicoro and Sifuentes-Osorio, 1984); 3. C. bifermantans in a 22 year old man with aortic and mitral valve prostheses who underwent thrombectomy of both valves and received 5 weeks of antibiotics and survived (Kolander et al., 1989) and 4. C. clostridiformis in a 71 year old man with a prosthetic aortic valve who survived without any cardiac intervention and a prolonged antibiotic course after splenectomy for a splenic abscess (Robles et al., 1997).

Clostridial spp. are generally susceptible to most antibiotics used for the treatment of anaerobic infections, including beta-lactam antibiotics, macrolides, imidazoles and chloramphenicol. Increased resistance of clostridia to several anti-microbial agents has been reported. Thus, the susceptibility of Clostridium spp. needs to be verified to ensure correct therapy. The management of clostridial infections often entails a combination of antimicrobial therapy and surgical intervention. This was the case with our patient, whose clinical picture did not stabilize until he underwent valve replacement surgery followed by another course of prolonged antibiotics.

\section{Conclusion}

Our case of prosthetic valve endocarditis, is the first description of Clostridium limosum monomicrobial infection. Organisms causing prosthetic valve endocarditis often lead to additional valve replacement surgery. This patient is the first to survive infection with $C$. limosum, only after valve replacement surgery and a subsequent prolonged course of antibiotic treatment.

\section{Funding Information}

No funding was received for this study.

\section{Author's Contributions}

All authors have contributed significantly to the preparation and writing of this manuscript.

Lok Yung: Participated in drafting and design of the manuscript.

Carl Urban: Contributed to drafting, data analysis and writing of the manuscript.

Negin Nikham: Contributed to data analysis and writing of the manuscript.

Glenn Turett: Coordinated the drafting, design and writing of the manuscript.

\section{Conflict of Interest}

The authors declare that they have no conflicts of interest.

\section{References}

Alvarez-Eicoro, S. and J. Sifuentes-Osorio, 1984. Bacteremia in prosthetic valve endocarditis; Diagnosis by blood smear. Arch. Int. Med., 144: 849-850. DOI: $10.1001 /$ archinte.144.4.849

Barth, H., S. Fischer, A. Moglish and C. Fortsch, 2015. Clostridial C3 toxins target monocytes/macrophages and modulate their functions. Front. Immunol., 6: 1-6. DOI: 10.3389/fimmu.2015.00339

Bistrom, M., A.M. Moisander-Jylha, S. Heinikainen, K. Pelkola and M. Raunio-Saarnisto, 2016. Isolation of Clostridium limosum from an outbreak of metritis in farmed mink. Acta Vet. Scand., 58: 1-4. DOI: $10.1186 / \mathrm{s} 13028-016-0230-7$

Cato, E.P., C.S. Cummins and L.D.S. Smith, 1948. Clostridium limosum andré in Prévot 1948, 165 amended description and pathogenic characteristics. Int. J. Syst. Evolu. Microbiol., 20: 305-16.

DOI: $10.1099 / 00207713-20-3-305$ 
Felner, J.M. and V.R. Dowell, 1970. Anaerobic bacterial endocarditis. N. Engl. J. Med., 283: 1188-1192. DOI: 10.1056/NEJM197011262832203

Gorbach, S.L. and H. Thadepalli, 1975. Isolation of Clostridium in human infections: Evaluation of 114 cases. J. Infect. Dis., 131: S81-S85.

DOI: 10.1093/infdis/131.Supplement.S81

Gordon, G. and J.L. Axelrod, 1985. Prosthetic valve endocarditis caused by Pseudallescheria boydii and Clostridium limosum. Mycopathology, 89: 129-134. DOI: $10.1007 / \mathrm{BF} 00447020$

Kolander, S.A., E.M. Cosgrove and A. Molavi, 1989. Report of a case caused by Clostridium bifermentans and review of the literature. Arch. Int. Med., 149: 455-56. DOI: 10.1001/archinte.149.2.455
Robles, P., F. Garcia-Gallego, J. de Alba, J. Garcia and F.J. Dominguez et al., 1997. Endocarditis protesica y absceso esplénico causaados por Clostridium clostridiformis, Rev. Esp. Cardiol., 50: 360-362. DOI: $10.1016 / \mathrm{S} 0300-8932(97) 73235-4$

Shibuya, H., H. Terashi, S. Kurata, Y. Ishii and S. Takayasu et al., 1994. Gas gangrene following sacral pressure sores. J. Dermatol., 21: 518-23. DOI: $10.1111 /$ j.1346-8138.1994.tb01786.x 